\title{
Expectativas y concepciones de los estudiantes del MAES en la especialidad de Ciencias
}

\author{
Solís R., E ${ }^{1}$; Martín del Pozo, $\mathbf{R}^{2}$; Rivero G., A. ${ }^{1}$ y Porlán A., R. ${ }^{1}$ \\ ${ }^{1}$ Departamento de Didáctica de las Ciencias Experimentales y Sociales. Facultad de Ciencias de la \\ Educación. Universidad de Sevilla. 2. Departamento de Didáctica de las Ciencias. Facultad de Educación. \\ Universidad Complutense de Madrid.E-mail:esolis@us.es
}

Todos los autores son miembros del Grupo DIE (Didáctica e Investigación Escolar) integrado en la red IRES (Innovación y Renovación Escolar) ${ }^{1}$

[Recibido en enero de 2013, aceptado en abril de 2013]

Este trabajo forma parte de una investigación realizada con una muestra amplia de estudiantes del Máster Universitario en Formación de Profesorado de Educación Secundaria Obligatoria (MAES) en las especialidades de Ciencias, de la mayoría de las universidades públicas de Andalucía y en un grupo de la Universidad Complutense de Madrid, sobre sus expectativas y concepciones didácticas. Los resultados de un Cuestionario de Expectativas y del Inventario de Creencias Científicas y Pedagógicas (INPECIP) (Porlán, 1989), antes y después de realizar el Módulo Específico, muestran una escasa evolución tanto en sus expectativas como en sus concepciones iniciales.

Palabras clave: Máster de Secundaria; Formación inicial; Expectativas del profesorado; Creencias Científicas y Pedagógicas.

\section{MAES students expectations and conceptions of the Science specialty}

This project belongs to a research effort done among a big sample of MAES (Máster in Teacher Training Secondary Education) students specialized in science, from most of the public universities in Andalucía and a group of Universidad Complutense of Madrid. The research has been focus on students' expectations and teaching conceptions. The results of a questionnaire about scientific and educational beliefs (INPECIP, Porlán, 1989), before and after studying this specific master, shows a few evolution between this final expectations and perceptions and the ones they had before.

Keywords: Secondary Master; Initial Training,;Teacher Expectations; Scientific and Pedagogical Beliefs.

\section{Introducción}

Durante el curso 2009-2010, en las universidades españolas se desarrolla por primera vez el Máster Universitario en Formación de Profesorado de Educación Secundaria Obligatoria y Bachillerato, Formación Profesional y Enseñanzas de Idiomas (MAES).

El proceso del diseño concreto del contenido de este MAES en las diferentes universidades ha sido muy diverso: desde el resultado de un proceso de negociación entre las distintas facultades y departamentos universitarios, como en la Universitat Autónoma de Barcelona (Deulofeu, Márquez y Sanmartí, 2010), hasta el clásico mecanismo de reparto de créditos, en el que los rectorados han pretendido responder a demandas, sin fundamento didáctico, de diversas facultades y departamentos, muchos de ellos sin relación alguna con la educación, como en algunas universidades de Andalucía (García, Solís y Porlán, 2010; González, Jiménez y Pérez, 2011) o en la Universidad de Santiago de Compostela (Trillo y Rodríguez, 2011).

Uno de los primeros estudios llevados a cabo sobre el MAES ha sido realizado por la Asociación de Profesores e Investigadores en Ciencias Experimentales, APICE, (Benarroch, 2011), mediante un cuestionario elaborado ad hoc y cumplimentado por 20 profesores de 14

\footnotetext{
${ }^{1}$ http://www.redires.net/
} 
universidades españolas. Se destacan algunas fortalezas del proceso de implantación del MAES tales como: a) valoración positiva del MAES respecto al CAP, b) fortalecimiento de las relaciones con los centros de secundaria, c) actitud y participación del profesorado, de los estudiantes y de la coordinación del Máster, d) utilización de entornos virtuales, y e) satisfacción con la asignatura Enseñanza y Aprendizaje de la Física y la Química.

De la misma manera destacan debilidades como: a) la ausencia de coordinación y de convenios que regulen las relaciones entre instituciones y entre el profesorado implicado en sus enseñanzas, y b) los sistemas de selección y reconocimiento del profesorado, sin tasar la experiencia docente y/o investigadora en la Educación Secundaria.

También, cabe resaltar el primer informe realizado por La Agencia Nacional de Evaluación de la Calidad y Acreditación, ANECA (2012), que entre sus conclusiones destaca, refiriéndose a las especialidades de Ciencias, que:

"Es muy importante que se evite la sobreespecialización en contenidos cientificos que reflejan muchas de las quejas recogidas entre el alumnado: la repetición de lo estudiado durante la carrera que acaban de finalizar, muchas veces impartido por los mismos profesores y con idénticos o similares contenidos. Los complementos siempre deben estar referidos a las materias de secundaria propias de la especialidad docente, no de la especialidad académica universitaria" (p. 23).

Finalmente, en el estudio que origina este trabajo ${ }^{2}$, se han analizado las páginas web de 76 Universidades españolas (53 públicas, 23 privadas) para recabar información acerca de la implantación del MAES (Solís, Martín del Pozo y Rivero, 2012). El grado de implantación del Máster en el curso 2010-2011 es alto (85\% de las universidades) y un $26 \%$ de las universidades que implantan el MAES no tienen Facultad de Ciencias de la Educación sobre todo de titularidad privada o privada de la Iglesia (en 10 de las 17 analizadas, es decir un 59 $\%)$.

\section{El estudio y la muestra}

El estudio se realizó en las universidades públicas andaluzas (a excepción de la universidad de Cádiz y de la Universidad Pablo Olavide de Sevilla) y también en un grupo de la Universidad Complutense de $\mathrm{Madrid}^{3}$, durante el curso 2010-2011. Todos los grupos estudiados eran de las especialidades de Ciencias, Física y Química y/o Biología y Geología, dependiendo de las universidades. El estudio se ha realizado al comienzo del Módulo Específico y al final del mismo (excepto en la Universidad de Córdoba que solo se realizó al inicio).

El estudio realizado tenía una doble finalidad ya que se han abordado dos problemas diferenciados, además de obtener una serie de datos para la contextualización de la muestra. Por una parte se trataba de recoger cuáles eran las expectativas de los estudiantes del MAES, antes de comenzar el Módulo Específico del mismo y que ha ocurrido con dichas expectativas al terminar dicho Módulo. Para ello se utilizó un cuestionario de expectativas que se muestra al hilo de los resultados obtenidos. El otro problema era el indagar acerca de sus creencias sobre Aprendizaje Científico, Naturaleza de la Ciencia, Metodología del Profesor y Modelos Didácticos, dimensiones todas ellas incluidas en el cuestionario del INPECIP, que se encuentra junto con sus resultados en el Anexo-I.

\footnotetext{
${ }^{2}$ Estudio realizado mediante un proyecto de investigación acogido a la concesión de una licencia por estudios a uno de los autores, por parte de la Consejería de Educación de la Junta de Andalucía.

${ }^{3}$ Este trabajo ha sido posible gracias a la desinteresada colaboración de: $\mathrm{M}^{\mathrm{a}}$ Rut Jiménez (UAL), Alfonso Pontes (UCO), Francisco Javier Perales (UGR), Ana Wamba y Ma Ángeles de las Heras (UHU), Rocío Quijano (UJAEN), Ángel Blanco (UMA) y Ángel Ezquerra (UCM).
} 
Los datos que caracterizan a la muestra de 190 estudiantes al inicio del módulo se refieren a las siguientes variables: edad, sexo, año de finalización de los estudios, si se ha tenido contacto previo con la docencia, si se trabaja actualmente y si se piensa dedicar a ejercer la docencia en Educación Secundaria.

Se ha realizado un análisis de frecuencias, medias y desviación típica, además de un análisis de contingencia para determinar si estos datos generales eran independientes o no de la universidad donde se realizaba el MAES. Los valores de Chi-cuadrado de Pearson $(\geq 0,005)$ indican que no existe dependencia entre la universidad donde se desarrolla el MAES y las variables consideradas.

De acuerdo con esto, las características de la muestra son las siguientes:

a) La edad media ponderada es de 26 años, abarca un abanico de 22 a 57 años, aunque el $90 \%$ de la muestra está entre 23 y 33 años.

b) El $64.2 \%$ de los estudiantes son mujeres y el $35.8 \%$ hombres.

c) La mayoría (65\%) ha acabado sus estudios en los tres últimos cursos, antes del de comienzo del MAES. Solo el $5 \%$ los ha concluido antes del año 2000.

d) El $84.7 \%$ son egresados de las universidades donde se han matriculado del MAES, aunque, la UGR es la que más alumnos aporta a toda la población del MAES de las universidades andaluzas estudiadas, ya que en sus grupos hay matriculados 30 alumnos y aporta 45. Como dato significativo, solamente hay un alumno procedente de una universidad extranjera matriculado en estas 8 universidades.

e) Los grupos eran todos de las especialidades del MAES de Física y Química, o de Biología y Geología. En concreto en la universidad de Huelva y Jaén, no había grupo de FQ y en la UCM no se pasó el cuestionario al grupo de BG. Las titulaciones mayoritarias son con diferencia, las licenciaturas de Biología, Química y Ciencias Medioambientales.

f) En el momento de realizar el MAES, un $63.7 \%$ declara que no trabaja y un $35.8 \%$ sí lo hace.

g) La mayoría (61.6\%) manifiesta que ha tenido contacto previo con la docencia, pero un porcentaje importante $(37.4 \%)$ no lo ha tenido.

h) El $63.55 \%$ de los que trabajan en algo relacionado con la docencia, indican que dan clases particulares y/o en academias.

i) Ante la posibilidad de dedicarse a la docencia en Secundaria, un $53.2 \%$ indica que sí, con toda probabilidad, y un $38.4 \%$ que es probable. Solo un $1.6 \%$ se lo tendría que pensar, mientras que un $6.8 \%$ no sabe o no contesta.

Según los valores de Chi-cuadrado de Pearson $(\leq 0,005)$, parece que la titulación, la universidad de titulación y el contacto previo con la docencia, mantienen una cierta relación de dependencia con la universidad donde se estudia el MAES.

Si comparamos algunos de estos datos con otros estudios realizados sobre estas mismas cuestiones, tanto en muestras de asistentes del anterior CAP o de asistentes al actual MAES, observamos que existen datos coincidentes en algunos de los aspectos estudiados.

Por ejemplo, en un estudio realizado en la Universidad de Jaén (Zagalaz-Sánchez et al., 2011), con alumnos del MAES de 10 especialidades (entre las que se encuentra Biología y Geología), coinciden en que las variables sexo y edad, no presentan dependencia con las variables que analizan las expectativas de los asistentes al MAES. 
También se obtienen datos similares con el CAP en las titulaciones mayoritarias, con la salvedad de la aparición en la actualidad de la licenciatura en Ciencias Medioambientales (Solís, 1998; Velázquez et al. 1999; Domínguez y Álvarez, 2001; García y Martínez, 2001 y Pro et al, 2001).

En cuanto a la posibilidad de dedicarse a la docencia en Secundaria, parece que los datos aquí recogidos difieren de estudios anteriores, en relación con el CAP (Solís, 1998; Córdoba, Ortega y Pontes, 2009), en el sentido de que en estos, el porcentaje de encuestados que manifestaban su interés por dedicarse a la docencia era menor que el que figura en este caso, que sumando los que piensan dedicarse con toda probabilidad o los que piensan que es muy probable, llegan a más del $91.6 \%$ de la muestra. Los mismos resultados se dan en el estudio anteriormente citado de Zagala-Sánchez et al. (2011), en el que es del 89.1\%.

Por último, en un estudio de Pontes et al. (2010), aunque también se analiza el interés por la docencia de los alumnos del CAP y del MAES de la Universidad de Córdoba (con una muestra referida a la totalidad de los alumnos del CAP y/o del MAES) no aparecen porcentajes, sino un valor de 3.07 en una escala de Likert de 1 a 5 . Es un valor bastante centrado, pero que no nos permite realizar comparaciones. Si indican las razones de este interés y el momento en que dicho interés se ha producido y, como ellos mismo indican, son bastante diversas y distintos. Datos similares se encuentran en otro estudio de Pontes et al. (2011).

En resumen, los estudiantes de este estudio tienen una edad media de 26 años, 2 de cada 3 son mujeres, que ha terminado su licenciatura en los tres años inmediatamente anteriores a su matrícula en el MAES, que ha realizado una titulación en Biología, Química o Ciencias Medioambientales y que se ha matriculado mayoritariamente en la universidad en la que realizaron su licenciatura. Dos de cada 3 dicen no trabajar en el momento de realizar el MAES y también 2 de cada 3 dicen que han tenido contacto previo con la docencia. Si trabajan en algo relacionado con la docencia, 2 de cada 3 también, lo hacen dando clases particulares o en academias. 5 de cada 10 piensan dedicarse con toda probabilidad a la docencia en Secundaria, aunque este número aumenta a casi 9 de cada 10 si incluimos los que indican que es posible.

\section{Instrumentos}

La parte del estudio que presentamos hace referencia a los datos obtenidos a partir de un cuestionario de expectativas y el Inventario de Creencias Pedagógicas y Científicas (Porlán, 1989). El número de cuestionarios cumplimentados se muestran en la Tabla 1.

Tabla 1. Número de cuestionarios por Universidad y Grupo.(Pre/Post).

\begin{tabular}{|l|l|l|l|l|l|l|l|l|}
\hline & UAL & UCO & UGR & UJAEN & UHU & UMA & US & UCM \\
\hline BG & $6 / 0$ & $16 / 0$ & $26 / 20$ & $14 / 9$ & $15 / 13$ & $19 / 19$ & $17 / 17$ & \\
\hline FQ & $8 / 8$ & $9 / 0$ & $10 / 7$ & & & $12 / 13$ & $19 / 18$ & $19 / 16$ \\
\hline Total & $14 / 8$ & $25 / 0$ & $36 / 27$ & $14 / 9$ & $15 / 13$ & $31 / 32$ & $36 / 35$ & $19 / 16$ \\
\hline \multicolumn{7}{|l|}{ Totales Pre : 171 Andalucía + 19 UCM $=190$. Totales post: 124 Andalucía + 16 UCM = 140 } \\
\hline
\end{tabular}

Los datos han sido analizados con el paquete informático PASW Statistics, Versión 18.0.0 (30 de julio de 2009) y el IBM SPSS Statistics, Versión 20.0.0 (2011). 


\section{El cuestionario de expectativas}

El cuestionario fue reelaborado a partir de otros trabajos previos (Solís, 1989) y se cumplimentó de forma anónima.

En el cuestionario inicial, las preguntas realizadas estaban organizadas en torno a cuatro apartados: razones para cursar el MAES, referencias al MAES anterior, expectativas sobre el desarrollo de las clases y los logros al final del MAES. Al final del Módulo Específico, se les volvía a interrogar sobre el desarrollo de las clases y sus logros. Las respuestas se cumplimentaban en una escala Likert desde el 1 (en completo desacuerdo) al 5 (en completo acuerdo). Los ítems correspondientes aparecen junto a las tablas de resultados.

Para determinar la fiabilidad y consistencia interna del cuestionario de expectativas, se calculó el valor del $\alpha$ de Cronbach. El resultado fue de 0.707 que, de acuerdo con distintos estudios es considerado suficiente (Cortina, 1993; Streiner, 2003; Oviedo y Campo-Arias, 2005).

\section{El Inventario de Creencias Pedagógicas y Científicas (INPECIP)}

El otro instrumento el INPECIP (Porlán, 1989), ya ha sido utilizado en otras investigaciones (Martín del Pozo, 1994; Solís y Porlán, 2003; Porlán y Martín del Pozo, 2004), se compone de 56 ítems, 14 por cada una de las cuatro dimensiones o categoría antes citadas.

Con una escala tipo Likert desde el 1 (en completo desacuerdo) al 5 (en completo acuerdo) los estudiantes manifestaron su grado de acuerdo con las declaraciones. Para determinar la fiabilidad y consistencia interna del INPECIP (aunque ya podemos considerarlo suficientemente validado por los estudios señalados), se calculó el valor la $\alpha$ de Cronbach, resultando ser 0.831, que se encuentra dentro de los parámetros aceptados, ya que es superior al 0.7 e inferior al 0.9, valor por encima del cual, se podría considerar que existen proposiciones redundantes en el cuestionario. Las dimensiones que aborda el cuestionario, como ya hemos indicado al definir, los problemas del estudio, son las siguientes: Aprendizaje científico, Naturaleza de las Ciencias, Modelo didáctico y Metodología del profesor. El INPECIP, con las declaraciones separadas por categorías y con los resultados correspondientes, se encuentra en el Anexo I.

El marco desde el cual hemos analizado los resultados del INPECIP está ligado a los presupuestos del Grupo DIE de la red IRES, ya explicitado en la literatura (Azcárate, 1999; Martín del Pozo, 1999; Porlán 1999; García, 1999; Porlán y Rivero, 1998; Garcia P., 2000; Martín del Pozo y Rivero, 2001; Porlán y Rivero, 2001; Rivero, 2003; Solís, 2005; Porlán et al., 2010 y Porlán et al., 2011). El instrumento básico de análisis utilizado es el modelo didáctico (Garcia, 2000), que se concibe como un instrumento de análisis de la realidad con la idea de intentar transformarla. Existe una tipificación de estos modelos, atendiendo a categorías relacionadas con los Problemas Prácticos Profesionales (Martín del Pozo y Rivero, 2001; Porlán y Rivero, 2001; Rivero, 2003; Solís y Porlán 2003; Solís, 2005; Porlán et al., 2010; Porlán et al., 2011), y una posible hipótesis de progresión entre unos modelos y otros, que no tiene por qué ser necesariamente lineal. Desde un modelo más tradicional o transmisivo (MDTR) hasta un modelo didáctico alternativo (Modelo de Investigación en la Escuela) (MIE), que consideramos como referente, con unos estadios intermedios en los llamados modelo didáctico tecnológico. (MDTC) y modelo didáctico activista o espontanéista (MDES). Las características de estos modelos se incluyen en el Anexo II (Solís, Porlán y Rivero 2012). 


\section{Presentación y discusión de los resultados}

\section{Las expectativas de los estudiantes}

En la Tabla 2 indicamos los resultados del cuestionario de expectativas antes de empezar el Módulo Específico.

Tabla 2. Resultado cuestionario de expectativas antes del Módulo Específico.

\begin{tabular}{|c|c|c|}
\hline Razones por las que te matriculas en este curso & Media & D. Tip \\
\hline $\begin{array}{l}\text { Me he matriculado en este máster porque lo exigen para presentarse a las } \\
\text { oposiciones. }\end{array}$ & 4,51 & ,842 \\
\hline $\begin{array}{l}\text { Me he matriculado en este máster porque me puede facilitar la superación de } \\
\text { dichas oposiciones. }\end{array}$ & 3,19 & 1,181 \\
\hline $\begin{array}{l}\text { Me he matriculado en este máster porque creo que me puede servir para mi } \\
\text { futuro profesional como profesor o profesora. }\end{array}$ & 3,79 & 1,022 \\
\hline $\begin{array}{l}\text { Me he matriculado en este máster porque es algo más que incorporar a mi } \\
\text { "curriculum vitae". }\end{array}$ & 3,26 & 1,310 \\
\hline Me he matriculado en este máster porque "nunca se sabe..." & 2,69 & 1,478 \\
\hline Referencias sobre el máster del curso anterior. & Media & D. Tip \\
\hline Resultó una pérdida de tiempo. & 3,14 & 1,130 \\
\hline Fue útil para las oposiciones. & 2,97 & 1,052 \\
\hline Fue útil para el futuro profesional de los alumnos y alumnas. & 2,91 & 0,947 \\
\hline Fue válido como experiencia personal y humana. & 2,99 & 0,96 \\
\hline $\begin{array}{l}\text { Referencias diversas dependiendo de los módulos y de los profesores y } \\
\text { profesoras que los impartieron. }\end{array}$ & 3,25 & 0,91 \\
\hline Las clases de este módulo deberían desarrollarse & Media & D. Tip \\
\hline Mediante explicaciones del profesor/a & 3,51 & 1,012 \\
\hline Trabajando individualmente los documentos y la información recibida & 2,93 & 1,118 \\
\hline $\begin{array}{l}\text { Trabajando en pequeño grupo y en gran grupo los documentos y la } \\
\text { información recibida. }\end{array}$ & 3,97 & 0,83 \\
\hline Debatiendo las propuestas que se puedan presentar. & 4,13 & 0,752 \\
\hline Dependiendo del tema se debe usar una u otra metodología & 4,34 & 0,785 \\
\hline Cuando finalice este Módulo Específico, creo que... & Media & D. Tip \\
\hline Estaré preparado/a para impartir clases en Enseñanza Secundaria & 3,12 & 0,960 \\
\hline Tendré una idea general de la didáctica específica de mi área. & 3,71 & 0 \\
\hline $\begin{array}{l}\text { Habré realizado una revisión y/o actualización de mis conocimientos } \\
\text { científicos }\end{array}$ & 3,24 & 1,089 \\
\hline Sabré programar las asignaturas de mi área en la Enseñanza Secundaria & 3,58 & 0,862 \\
\hline $\begin{array}{l}\text { Tendré una idea general acerca de cómo se lleva a cabo la investigación en la } \\
\text { didáctica de mi especialidad }\end{array}$ & 3,59 & 0,829 \\
\hline
\end{tabular}

a) Con respecto a las razones para cursar el Máster, la respuesta que mayor grado de acuerdo consigue, muy próximo al completamente de acuerdo, es aquella que relaciona la obligatoriedad del MAES con las oposiciones. Estas razones están relacionadas con la edad, el año de finalización de los estudios, el contacto previo con la docencia y la universidad donde estudian el MAES. Cuanto más se aproxima el año de finalización de los estudios a la actualidad, los valores obtenidos son menores que lo esperado en cuanto al estar de acuerdo con la afirmación que recoge la declaración, variación que coincide con los datos obtenidos a edades menores y con aquellos que dicen haber tenido contacto previo con la docencia. En cuento a la universidad de titulación, los titulados por la UCM se declaran menos de acuerdo de lo esperado con esa afirmación y los de la UJAEN más de acuerdo de lo esperado. 
b) Los estudiantes no tienen una información suficiente como para hacer una valoración del $M A E S$ del curso anterior, por lo que sus respuestas se sitúan alrededor de la zona insegura, no existiendo ninguna declaración que presente relaciones de dependencia con otras variables.

c) De las propuestas metodológicas que se les plantean, están de acuerdo en que, según el tema, se puede usar una u otra metodología. El trabajo en pequeño grupo y el debate también obtienen un amplio acuerdo. La propuesta de explicaciones del profesor, aunque se acerca a la zona insegura se encuentra a medio camino entre dicha inseguridad y el acuerdo. La que parece despertar menos acuerdo es la idea del trabajo individual de la información que puedan recibir. En este apartado no existe ninguna declaración que presente relaciones de dependencia con otras variables.

d) Respecto a las expectativas de aprendiaaje al final del Módulo Específico, casi todas las respuestas se mueven en el terreno de la inseguridad y con desviaciones típicas bastante parecidas. Quizás la afirmación de que tendrán una idea general de la didáctica específica del área, es la que más se aproxima al acuerdo. En este aspecto es donde más relaciones se detectan con otras variables. Por ejemplo, los que consideran que cuando acaben el Módulo estarán preparados para impartir clases en Secundaria, son menos que los que cabría esperar en relación con que seguramente se dedicarán a la docencia en Secundaria. También parece que los hombres se muestran menos de acuerdo de lo previsto que las mujeres, en relación con considerar que van a tener una idea general de la didáctica específica de la materia. Asimismo los encuestados que manifiestan haber impartido clases particulares se sienten más seguros de lo esperado. De la misma manera muestran más seguridad las titulaciones que podemos considerar preferentes en este MAES, es decir: Biología, Física, Ciencias Medioambientales y Química, siendo la licenciatura en Geología la excepción en este sentido.

¿Qué ha resultado de las expectativas manifestadas respecto al Módulo Específico? En la Tabla 3, se indican los resultados de las expectativas después del Módulo Específico.

Tabla 3. Resultado cuestionario de expectativas después del Módulo Específico.

\begin{tabular}{|l|c|c|}
\hline Las clases de este módulo se han desarrollado: & Media & D. Tip \\
\hline Mediante explicaciones del profesor/a & 3,29 & 1,108 \\
\hline Trabajando individualmente los documentos y la información recibida & 3,01 & 1,025 \\
\hline $\begin{array}{l}\text { Trabajando en pequeño grupo y en gran grupo los documentos y la información } \\
\text { recibida. }\end{array}$ & 3,92 & 0,922 \\
\hline Debatiendo las propuestas que se puedan presentar. & 3,69 & 1,141 \\
\hline Dependiendo del tema se debe usar una u otra metodología & 3,64 & 1,129 \\
\hline Ahora que he finalizado este Módulo Específico, creo que... & Media & D. Tip \\
\hline Estoy preparado/a para impartir clases en Enseñanza Secundaria & 3,10 & 1,237 \\
\hline Tengo una idea general de la didáctica específica de mi área. & 3,48 & 1,035 \\
\hline He realizado una revisión y/o actualización de mis conocimientos científicos & 2,91 & 1,144 \\
\hline Sé programar las asignaturas de mi área en la Enseñanza Secundaria & 2,94 & 1,104 \\
\hline $\begin{array}{l}\text { Tengo una idea general acerca de cómo se lleva a cabo la investigación en la } \\
\text { didáctica de mi especialidad }\end{array}$ & 3,25 & 1,074 \\
\hline
\end{tabular}

a) En relación con la metodología desarrollada en el MAES, las expectativas parecen haberse cumplido, de forma más o menos general, en lo que se refiere a las explicaciones del profesor o profesora, al desarrollo del trabajo individual y a trabajar en pequeño y en gran grupo. En el 
aspecto de si las clases se han desarrollado mediante explicaciones del profesor/a, en todas las universidades analizadas se dan diferencias entre las frecuencias esperadas y las realmente observadas. En unos casos, las frecuencias observadas se aproximan más al desacuerdo con esta afirmación y en otras más próximas al acuerdo. Lo mismo ocurre en relación con el ítem del trabajo en grupo y con el debate de propuestas. Si resulta llamativo que esta situación de dependencia se da con respecto a la universidad donde se desarrolla el MAES, pero es independiente de si el grupo es de FQ o BG dentro de la misma universidad, situación que se da en UGR, UMA y US.

b) En relación con la preparación adquirida al final del Módulo Específico, las variaciones en este caso no se pueden generalizar, aunque en todas las declaraciones se produce un desplazamiento hacia la zona de inseguridad en el post. Los valores están prácticamente en el centro de la escala, salvo que en el pre, el valor de la desviación típica era menor que en el post, lo cual indica que la dispersión aumenta. Quizás uno de los aspectos más llamativos es el descenso hacia la zona de inseguridad de la programación de las asignaturas del área. En esta declaración si aparece una dependencia con la universidad del MAES y la universidad de titulación. En el primer caso, las frecuencias observadas unas se aproximan más al desacuerdo con esta afirmación y en otras más próximas al acuerdo. Respecto a la universidad de titulación ocurre lo mismo, aunque resulta llamativo que coincidan en ambos casos y sean las mismas universidades (las de titulación y las de estudio del MAES). No hay dependencia con ninguna de las otras 14 universidades de titulación recogidas.

Otros estudios realizados sobre expectativas de los asistentes a los cursos del MAES (Zagala et al, 2011), son coincidentes en aspectos como que los estudiantes prefieren los métodos y vías de aprendizaje en donde ellos tienen un papel activo en el proceso.

\section{Las concepciones de los estudiantes}

En primer lugar destacar que a la hora de analizar los datos, hemos suprimido los datos correspondientes a la UCO, ya que aparecían en el pre y no el post. Para realizar el análisis y discusión de los datos, lo haremos revisando cada una de las categorías y, dentro de ellas las declaraciones o proposiciones que tienen relación entre sí y que consideramos más representativas. Como pautas para este análisis, el desacuerdo se sitúa entre valores medios desde el 1.0 al 2.4. Admitiremos una zona de mayor o menor inseguridad, entre el 2.5 y el 3.4. El acuerdo se situará entre el 3.5 y el 5.0. Para considerar cambios, tomaremos una diferencia de 0.5 en el valor de la media. En el Anexo I se indican los resultados de cada una de las categorías estudiadas.

\section{Aprendiraje cientifico}

a) Antes de iniciar el Módulo Especifico. En esta categoría su opinión sobre la existencia de las ideas de los alumnos/as y su utilización didáctica, se sitúan en un MDTC y/o MDES, como modelos intermedios, ya que se encuentra en valores próximos a la inseguridad, pero por encima, es decir, en el límite inferior de lo que hemos considerado acuerdo, aunque también las consideran como errores conceptuales que es necesario sustituir por el conocimiento riguroso. En referencia a los requisitos para que se produzca aprendizaje, los podríamos situar en una mezcla de los MDTC, MDES e incluso MIE. Esta afirmación se ve corroborada por sus acuerdos respecto a cuándo consideran que un aprendizaje es significativo. Finalmente, sobre los tipos de contenidos que debe abarcar el aprendizaje científico, podríamos decir que cuando las declaraciones están más relacionas con las ideas o los presupuestos teóricos (saberes disciplinares, epistemológicos, etc.), sus consideraciones sobre el aprendizaje científico, se encuentran más evolucionados hacia modelos alejados del tradicional o 
transmisivo (tecnológico, espontaneísta e incluso alternativo). Como contraste, cuando las manifestaciones están más relacionadas con lo que puede ser el terreno de la práctica (saberes prácticos o experienciales) o de la aplicación en el aula de las ideas y teorías se manifiestan más próximos a dicho modelo tradicional.

b) ¿Se han producido cambios después del desarrollo del Módulo Especifico? Como se puede observar en el Anexo I, nos encontramos con que se han producido algunos cambios que, consideramos que alejan ciertas concepciones de los modelos más tradicionales y evolucionan hacia modelos intermedios e incluso a nuestro modelo de referencia. Es el caso de las declaraciones 5 y 46 relacionadas con las ideas de los alumnos y su uso didáctico, el 24 y el 27 relacionadas con la comprensión de los conceptos y el 35 y 48 relacionadas con las condiciones del aprendizaje. En todos estos casos hay un desplazamiento hacia posiciones menos tradicionales.

\section{Naturaleza de las Ciencias}

a) Antes de iniciar el Módulo Específico. En el análisis de esta categoría, los acuerdos manifestados por los estudiantes del MAES, se encuentran bastante dispersos y se mueven alternativamente desde el extremo de lo que podríamos denominar racionalismo empirista, más próximas a concepciones relacionadas con un MDTR, hacia posiciones mucho más relativistas y constructivistas, relacionadas con un MIE. Es decir, existe una especie de continuo entre una imagen rígida y dogmática de la ciencia y su evolución histórica y una visión más integradora, creativa y humana de la misma, sin que ninguno de esos dos extremos prevalezca, en nuestra opinión, sobre el otro. Es decir, una cierta indefinición epistemológica.

b) ¿Se han producido cambios después del desarrollo del Módulo Especifico? Como se puede observar en el Anexo I, podríamos decir que con ese margen de diferencia de 0.5 puntos, no ha habido cambios en las respuestas del cuestionario. Si consideramos aquellos ítems que se aproximan a esa variación nos encontramos con las declaraciones 4, 40 y 47 que muestran una cierta tendencia al cuestionamiento del método científico como garante de la objetividad y efectividad de la investigación científica y a dudar sobre si la evolución de la ciencia a lo largo de la historia es un proceso acumulativo o de construcción social del conocimiento.

\section{Modelo Didáctico}

a) Antes de iniciar el Módulo Específico. En esta categoría las manifestaciones, se ubican y polarizan en distintos modelos didácticos según se refiera a unos aspectos u otros. Hay declaraciones que implicarían un posicionamiento próximo al MIE y otras en las que se sitúan, en lo que podemos considerar, el otro extremo, el MDTR, existiendo toda una gama de situaciones híbridas e intermedias. Este entramado de manifestaciones nos lleva a pensar que no existe en la muestra, como no puede ser de otra manera, una definición precisa del modelo didáctico en el que ubicar a los sujetos de la misma. Dependiendo de la declaración de que se trate, éstos, se pueden ubicar en uno u otro modelo, incidiendo en la imposibilidad de encontrar entre el profesorado modelos didácticos "puros".

b) ¿Se han producido cambios después del desarrollo del Módulo Especifico? Como se puede observar en el Anexo I, prácticamente no se aprecian cambios significativos en este categoría, antes y después de la impartición del Módulo Específico del MAES. De las declaraciones relacionadas con el Modelo Didáctico, solamente dos presentan un cambio significativo, el 30 y el 34. En el primero se desplazan desde la franja mínima del acuerdo a la zona de inseguridad, en relación a que el trabajo en el aula debe estar centrados en los contenidos del área y aumenta su inseguridad, llegando casi a la zona del desacuerdo cuando se pronuncian acerca de que el libro de texto es un recurso indispensable para la enseñanza de las ciencias. 


\section{Metodología del profesor}

a) Antes de iniciar el Módulo Específico. En esta categoría, un considerable número de declaraciones (9 sobre 14 de las que componen esta categoría), obtienen unos valores que se encuentran en la zona intermedia de la escala, es decir, lo que hemos denominado la zona insegura. En lo que se refiere a la metodología en general, se manifiestan inseguros, pero con cierta tendencia al acuerdo en cuanto a reducir el método de enseñanza únicamente a la manera de dar los contenidos científicos. Asimismo se encuentran en la frontera entre la inseguridad y el acuerdo con la declaración de que cada profesor/a construye su propia metodología de enseñanza de las ciencias. En relación con la exposición magistral del profesor, se muestran de acuerdo en que es necesario explicar detenidamente los temas para facilitar el aprendizaje de las ciencias y se muestran inseguros en que la enseñanza de las ciencias basada en la explicación verbal favorezca que el alumno/a memorice mecánicamente el contenido. En lo que se refiere a la utilización del libro de texto, se muestran inseguros al afirmar que la mayoría de los libros de texto no facilitan la comprensión, el aprendizaje y la motivación del alumnado.

b) ¿Se han producido cambios después del desarrollo del Módulo Específico? En esta categoría vuelven a ser mínimos los cambios después del desarrollo del Módulo Específico. De acuerdo con el criterio de cambios que hemos venido utilizando, solamente la declaración 43 , relacionado con las exposiciones magistrales, es decir con una visión tradicional de la enseñanza, se pasa prácticamente del acuerdo a la zona de inseguridad. Hay otras dos declaraciones el 1 y el 9, muy relacionadas con una visión tecnológica de la enseñanza de las ciencias, en el que hay un mínimo cambio en el nivel de acuerdo que disminuye (en la 1) y un aumento de la inseguridad tendente a la zona de desacuerdo (en la 9).

Los resultados aquí obtenidos, en algunos casos no difieren de otros recogidos en estudios con profesorado en ejercicio o en formación inicial (Porlán, 1989; Martín del Pozo, 1994 y Solís y Porlán, 2003). Cabe destacar que en este último caso que se llevó a cabo un estudio similar con alumnos del CAP de la Universidad de Sevilla del curso 1997/98, en la especialidad de Física y Química, con una muestra de tamaño similar, los resultados obtenidos entonces, son bastante similares a los obtenidos en el cuestionario pasado antes del inicio del Módulo Específico. Los datos son prácticamente iguales en la categoría de Naturaleza de la Ciencia y Metodología del Profesor. Difieren en algunos ítems de la de Modelo Didáctico y donde presenta más variaciones es en la Aprendizaje Científico.

\section{Conclusiones}

Los estudiantes se han matriculado en el MAES porque es un requisito para presentarse a las oposiciones y en cierta medida porque les puede servir para su futuro profesional. La edad, el año de finalización de los estudios y su contacto previo con la docencia, son variables que se relacionan con esta afirmación.

¿Cuáles son sus expectativas? Esperan encontrar en el MAES una metodología que dependerá del tema a tratar, pero esperan trabajar en grupo y realizar debates. Una vez finalizado el Módulo Específico, todas las contestaciones se desplazan hacia la zona de inseguridad, manteniéndose prácticamente en los valores iniciales el trabajo en grupo y el trabajo individual. Sobre las posibles competencias desarrolladas en el Módulo Específico sus valoraciones se desplazan hacia la zona de inseguridad, independientemente de la universidad donde se ha desarrollado el MAES, excepto en lo que consideran cuál es su preparación para la programación de asignaturas del área. 
¿Cuáles son sus concepciones? Existen bastantes contradicciones en las manifestaciones de esta muestra de asistentes al MAES en las universidades estudiadas, en las dimensiones que componen el cuestionario del INPECIP. En el aprendizaje científico sus concepciones más teóricas parecen estar más evolucionadas hacia modelos didácticos más alternativos, que cuando se trata de llevar esas concepciones al terreno de la práctica. Presentan una indefinición epistemológica en relación con la naturaleza de las ciencias. Los modelos didácticos en los que se pueden ubicar están más en función de sus respuestas a proposiciones concretas que en el hecho de conformar una concepción coherente. $\mathrm{Y}$ en cuanto a la metodología del profesor, aunque también presentan una cierta indefinición, si mantienen declaraciones que los hacen estar más próximos a un modelo didáctico tradicional y/o transmisivo.

¿Han evolucionado sus concepciones? Los cambios producidos después del desarrollo del Módulo Específico son bastante reducidos. La categoría en que estos cambios son algo más significativos es en la de Aprendizaje Científico, mientras que en las otras tres los cambios son apenas perceptibles.

Excede del objeto de este trabajo el determinar las razones de este tipo de evolución. No obstante pensamos que sería interesante, a partir de estos datos, otro tipo de análisis, como sería el estudio de la posible relación entre los cambios y los aspectos que permanecen sin alterar y las asignaturas impartidas en el Módulo Específico, la titulación, la universidad donde se ha estudiado el MAES, etc., todo con la idea de que como indican Vilches y Gil (2010), el MAES contribuya a una verdadera renovación de la enseñanza y no solamente como un paso necesario, pero no suficiente, para la incorporación de nuevos profesores y profesoras al sistema educativo.

\section{Referencias bibliográficas}

ANECA (2012). Sobre la implantación del Máster Universitario de Formación del Profesorado de Educación Secundaria Obligatoria, Bachillerato, Formación Profesional y Enseñanza de Idiomas, análisis de situación y propuestas de mejora. http://es.scribd.com/doc/88012428/InformeMaster-Secundaria-ANECA (Consultado 23 de febrero de 2012)

Azcárate Goded, P. (1999). Metodología de enseñanza. Cuadernos de Pedagogía, 276, 72-78.

Benarroch, A.; Asociación de Profesores e Investigadores en Ciencias Experimentales (2011). Diseño y desarrollo del máster en profesorado de educación secundaria durante su primer año de implantación. Revista Eureka sobre Enseñanza y Divulgación de las Ciencias, 8 (1), 20-40. En línea en: http://hdl.handle.net/10498/10203

Córdoba, F., Ortega, R. y Pontes, A. (2009). Universitarios de ciencias ante la docencia en educación secundaria como expectativa profesional. Revista de Educación, 348, 401-421.

Cortina. J. M. (1993). What Is Coefficient Alpha? An Examination of Theory and Applications. Journal of Applied Psychology, 78 (1), 98-104.

Deulofeu, J., Márquez, C. y Sanmartí, N. (2010). Formar profesores de secundaria. La experiencia de la Universitat Autónoma de Barcelona. Cuadernos de Pedagogía, 404, 8084.

Domínguez C., J. M. y Álvarez P., V., (2001). La formación inicial de profesorado de Física y Química de Educación Secundaria en la Universidad de Santiago de Compostela. Revista Interuniversitaria de Formación del Profesorado, 40, 81-96.

García Díaz, J.E. (1999). Las ideas de los alumnos. Cuadernos de Pedagogía, 276, 58-64. 
García, S. y Martínez, C., (2001). Las ideas de los alumnos del CAP, punto de referencia para reflexionar sobre formación docente. Revista Interuniversitaria de Formación del Profesorado, 40, 97-110.

García, F. (2000) Los modelos didácticos como instrumento de análisis y de intervención en la realidad educativa. Revista Bibliográfica de Geografía y Ciencias Sociales no 205 Biblio 3W. Universidad de Barcelona (http://www.ub.es/geocrit/b3w-207.htm) (Consultado 17 de diciembre de 2012).

García, F., Solís, E. y Porlán, R. (2010). El máster en Sevilla: frustraciones y oportunidades. Cuadernos de Pedagogía, 404, 85-87.

González Faraco, J.C., Jiménez Vicioso, J.R. y Pérez Moreno, H.M. (2011). El nuevo modelo formativo del profesorado de Educación Secundaria y su proceso de implantación en las universidades Andaluzas. Fuentes, 11, 66-85.

Martín del Pozo, R. (1994). El conocimiento del cambio químico en la formación inicial del profesorado. Estudio de las concepciones disciplinares y didácticas de los estudiantes de magisterio. Tesis doctoral inédita. Universidad de Sevilla.

Martín del Pozo, R. (1999), Las materias escolares. Cuadernos de Pedagogía, 276, 58-64.

Martín del Pozo, R, y Rivero García, A., (2001). Construyendo un conocimiento profesionalizado para enseñar ciencias en la Educación Secundaria: los Ámbitos de Investigación Profesional en la formación inicial del profesorado. Revista Interuniversitaria de Formación del Profesorado, 40, 63-79.

Oviedo, H. y Campos-Arias, A. (2005). Aproximación al uso del coeficiente Alfa de Conbrach. Revista Colombiana de Psiquiatría, XXXIV (4), 572-580.

Pontes, A., Ariza, L. y Del Rey, R. (2011). Identidad profesional docente en aspirantes a profesorado de enseñanza secundaria. Psychology, Society, \& Education 2 (2), 131-142.

Pontes, A., Ariza, L., Serrano, R. y Sánchez, F.J. (2011). Interés por la docencia entre aspirantes a profesores de Ciencia y Tecnología. Revista Eureka sobre Enseñanza y Divulgación de las Ciencias 8 (2), 180-195. En línea en: http://hdl.handle.net/10498/10852

Porlán, R., (1989). Teoría del conocimiento, Teoría de la enseñanza y desarrollo profesional. Tesis Doctoral. Universidad de Sevilla.

Porlán, R. (1999). Formulación de contenidos escolares. Cuadernos de Pedagogía, 276, 65-70

Porlán, R. y Martín Del Pozo, R. (2004). The Conceptions of In-service and Prospective Primary School Teachers About the Teaching and Learning of Science. Journal of Science Teacher Education, 15(1), 39-62.

Porlán, R. y Rivero, A., (2001). Nature et organisation du savoir profesionnel enseignant "souhaitable". Aster, 32, 221-251.

Porlán, R. Y Rivero, A. (1998). El conocimiento de los profesores. Díada Editoras: Sevilla.

Porlán, R., Martín Del Pozo, R.; Rivero, A., Harres, J.; Azcárate, P. y Pizzato, M. (2010). El cambio del profesorado de ciencias I: marco teórico y formativo. Enseñanza de las Ciencias, 28(1), 31-46

Porlán, R., Martín Del Pozo, R.; Rivero, A., Harres, J.; Azcárate, P. y Pizzato, M. (2010). El cambio del profesorado de ciencias II: itinerarios de progresión y obstáculos en estudiantes de magisterio. Enseñanza de las Ciencias, 29(3), 413-426. 
Pro, A., Sánchez, G., Saura, O. y Valcárcel, Mª V., (2001). ¿Cómo es valorado un curso de formación inicial desde las necesidades y problemas de profesores principiantes? Revista Interuniversitaria de Formación del Profesorado, 40, 97-110.

Rivero G., A., (2003). Globalización e Investigación del Medio. Proyecto Docente. Universidad de Sevilla.

Solís, E., (1998). Análisis de las opiniones e impresiones de los asistentes a un Curso de Formación Inicial de Profesores de Secundaria. Investigación en la Escuela, 35, 87-98.

Solís, E. (2005). Concepciones Curriculares del Profesorado de Física y Química en Formación Inicial. Tesis doctoral. Universidad de Sevilla.

Solís, E. y Porlán, R., (2003). Las concepciones del profesorado de Ciencias de Secundaria en Formación Inicial ¿Obstáculo o punto de partida? Investigación en la Escuela, 49, 5-22.

Solís, E., Martín Del Pozo, R. y Rivero, A. (2012). Qué dicen esperar y qué dicen que han encontrado los estudiantes del MAES, en la especialidad de Ciencias. XXV Encuentros de Didáctica de las Ciencias Experimentales. Santiago de Compostela. 173-180.

Solís, E., Porlán, R. y Rivero, A. (2012). ¿Cómo representar el Conocimiento Curricular de los profesores de Ciencias y su evolución? Enseñanza de las Ciencias, 30 (3), 9-30.

Streiner, D. L. (2003). Starting at the Beginning: An Introduction to Coefficient Alpha and Internal Consistency. Journal of Personality Assessment, 80(1), 99-103

Trillo, F. y Rodríguez, X., (2011). La formación inicial del profesorado de enseñanza secundaria: crónica de una experiencia. El caso de la universidad de Santiago de Compostela en el contexto de Galicia. Fuentes, 11, 41-65

Velázquez, J., Azcárate, P., Ibarra, M. y Navarrete, A., (1999). La formación inicial del profesorado Educación Secundaria ante su nueva andadura. Evaluación de una experiencia piloto. Universidad de Cádiz.

Vilches, A. y Gil, D. (2010). Máster de formación inicial del profesorado de Enseñanza Secundaria. Algunos análisis y propuestas. Revista Eureka sobre Enseñanza y Divulgación de las Ciencias, 7 (3), 661-666. En línea en: http://hdl.handle.net/10498/9818

Zagalaz-Sánchez, M. L., Molero, D., Campoy-Aranda, T. J. y Cachón-Zagalaz, J. (2011). Las expectativas depositadas en el máster de Educación Secundaria por los futuros docentes durante su formación inicial. Revista de Investigación en Educación, 9 (2), 19-34. 
ANEXO I. Resultados del INPECIP

\begin{tabular}{|c|c|c|c|c|c|c|}
\hline Aprendizaje Científico & \multicolumn{3}{|c|}{ PRE MAES } & \multicolumn{3}{|c|}{ POST MAES } \\
\hline Declaraciones & $\mathrm{N}$ & $\overline{\bar{X}}$ & $\bar{\sigma}$ & $\mathrm{N}$ & $\overline{\bar{X}}$ & $\sigma$ \\
\hline $\begin{array}{l}\text { 5.- Las ideas espontáneas de los alumnos/alumnas } \\
\text { deberían ser el punto de partida para el aprendizaje } \\
\text { de contenidos científicos }\end{array}$ & 164 & 3,52 & 971 & 139 & 4,08 &, 975 \\
\hline $\begin{array}{l}\text { 8.- Un aprendizaje será significativo cuando el } \\
\text { alumno/alumna sea capaz de aplicarlo a } \\
\text { situaciones diferentes }\end{array}$ & 165 & 4,46 & 623 & 139 & 4,45 & ,630 \\
\hline $\begin{array}{l}\text { 14.- Los alumnos y alumnas suelen deformar } \\
\text { involuntariamente las explicaciones verbales de los } \\
\text { profesores y profesoras y la información que leen } \\
\text { en los libros de texto. }\end{array}$ & 165 & 3,65 & 894 & 138 & 3,75 & 854 \\
\hline $\begin{array}{l}\text { 19.- Los alumnos y alumnas no tienen capacidad } \\
\text { para elaborar espontáneamente, por ellos mismos, } \\
\text { concepciones acerca del mundo natural y social } \\
\text { que les rodea. }\end{array}$ & 165 & 2,07 & ,939 & 139 & 1,89 & ,944 \\
\hline $\begin{array}{l}\text { 24.- Cuando el profesor o profesora explica con } \\
\text { claridad un concepto científico, y el alumno o } \\
\text { alumna está atento, se produce aprendizaje. }\end{array}$ & 164 & 3,04 & 1,164 & 138 & 2,41 & 1,153 \\
\hline $\begin{array}{l}\text { 27.- Los aprendizajes científicos esenciales que } \\
\text { deben realizar los alumnos y alumnas en las aulas } \\
\text { son los relacionados con la comprensión de los } \\
\text { conceptos. }\end{array}$ & 163 & 3,48 & ,956 & 136 & 2,85 & 977 \\
\hline $\begin{array}{l}\text { 32.- Los alumnos y alumnas están más capacitados } \\
\text { para comprender un contenido si lo pueden } \\
\text { relacionar con conocimientos previos que ya } \\
\text { poseen. }\end{array}$ & 164 & 4,40 &, 714 & 136 & 4,11 & ,740 \\
\hline $\begin{array}{l}\text { 33.- El aprendizaje científico es significativo } \\
\text { cuando el alumno / alumna tiene un interés } \\
\text { personal relacionado con lo que aprende. }\end{array}$ & 164 & 3,94 & 1,001 & 137 & 3,66 & 1,007 \\
\hline $\begin{array}{l}\text { 35.- Para aprender un concepto científico es } \\
\text { necesario que el alumno o la alumna haga un } \\
\text { esfuerzo mental por grabarlo en su memoria. }\end{array}$ & 163 & 2,65 & 1,120 & 137 & 2,11 & 1,158 \\
\hline $\begin{array}{l}\text { 41.- Los alumnos y alumnas, cuando son capaces } \\
\text { de responder correctamente a las cuestiones que } \\
\text { les plantea el profesor o profesora, demuestran que } \\
\text { han aprendido. }\end{array}$ & 164 & 3,15 & 1,056 & 137 & 2,71 & 1,048 \\
\hline $\begin{array}{l}\text { 46.- Los errores conceptuales deben corregirse } \\
\text { explicando la interpretación correcta de los } \\
\text { mismos tantas veces como el alumno/alumna lo } \\
\text { necesite. }\end{array}$ & 164 & 3,91 & 977 & 135 & 3,30 & ,996 \\
\hline $\begin{array}{l}\text { 48.- En general, los alumnos/alumnas son más o } \\
\text { menos listos/ listas según las capacidades que } \\
\text { posean. }\end{array}$ & 162 & 2,72 & 1,045 & 137 & 2,28 & 1,035 \\
\hline $\begin{array}{l}\text { 50.- El aprendizaje científico de los alumnos / } \\
\text { alumnas no sólo debe abarcar datos o conceptos, } \\
\text { sino también, y al mismo tiempo, los procesos } \\
\text { característicos de la metodología científica } \\
\text { (observación, hipótesis, etc.) }\end{array}$ & 161 & 4,17 & ,677 & 136 & 4,13 & ,676 \\
\hline $\begin{array}{l}\text { 54.- Para que los alumnos/alumnas aprendan de } \\
\text { manera significativa es importante que se sientan } \\
\text { capaces de aprender por si mismos/mismas. }\end{array}$ & 163 & 4,21 & 696 & 137 & 4,33 & 698 \\
\hline Naturaleza de las Ciencias & & PRE M & & & OST 1 & \\
\hline
\end{tabular}




\begin{tabular}{|c|c|c|c|c|c|c|}
\hline Declaraciones & $\mathrm{N}$ & $\bar{X}$ & $\sigma$ & $\mathrm{N}$ & $\bar{X}$ & $\sigma$ \\
\hline $\begin{array}{l}\text { 4.- Las teorías científicas obtenidas al final de un } \\
\text { proceso metodológico riguroso, son un reflejo } \\
\text { cierto de la realidad. }\end{array}$ & 164 & 3,24 & ,908, & 138 & 2,85 & 1,060 \\
\hline $\begin{array}{l}\text { 11.- En la observación de la realidad es imposible } \\
\text { evitar un cierto grado de deformación que introduce } \\
\text { el observador. }\end{array}$ & 165 & 4,12 & 747, & 139 & 4,06 & 809, \\
\hline $\begin{array}{l}\text { 21.- El observador científico no debe actuar bajo la } \\
\text { influencia de teorías previas sobre el programa } \\
\text { investigado. }\end{array}$ & 164 & 2,96 & ,965 & 136 & 2,82 & 1,128 \\
\hline $\begin{array}{l}\text { 22.- Toda investigación científica comienza por la } \\
\text { observación sistemática del fenómeno que se } \\
\text { estudia. }\end{array}$ & 164 & 3,99 & 893 & 137 & 3,75 & 991 \\
\hline $\begin{array}{l}\text { 23.- El conocimiento humano es fruto de la } \\
\text { interacción entre el pensamiento y la realidad. }\end{array}$ & 160 & 4,06 & 795, & 136 & 3,96 & 744 \\
\hline $\begin{array}{l}\text { 28.- El pensamiento de los seres humanos está } \\
\text { condicionado por aspectos subjetivos y } \\
\text { emocionales. }\end{array}$ & 164 & 4,09 & 847, & 137 & 4,13 & ,756 \\
\hline $\begin{array}{l}\text { 38.- El investigador siempre está condicionado, en } \\
\text { su actividad, por las hipótesis que intuye acerca del } \\
\text { problema investigado. }\end{array}$ & 163 & 3,52 & 863, & 134 & 3,40 & 884, \\
\hline $\begin{array}{l}\text { 39.- El conocimiento científico se genera gracias a la } \\
\text { capacidad que tenemos los seres humanos para } \\
\text { plantearnos problemas e imaginar posibles } \\
\text { soluciones a los mismos. }\end{array}$ & 164 & 3,95 & ,769 & 137 & 3,91 & 812 \\
\hline $\begin{array}{l}\text { 40.- La eficacia y la objetividad del trabajo científico } \\
\text { estriba en seguir fielmente las fases ordenadas del } \\
\text { método científico: observación, hipótesis, } \\
\text { experimentación y elaboración de teorías. }\end{array}$ & 162 & 3,80 & 802 & 136 & 3,40 & 1,118 \\
\hline $\begin{array}{l}\text { 42.- La metodología científica garantiza totalmente } \\
\text { la objetividad en el estudio de la realidad. }\end{array}$ & 164 & 3,05 & ,922 & 135 & 2,73 & 1,018 \\
\hline $\begin{array}{l}\text { 44.- A través del experimento, el investigador } \\
\text { comprueba si su hipótesis de trabajo es verdadera o } \\
\text { falsa. }\end{array}$ & 163 & 4,17 & ,705 & 137 & 3,94 & 838, \\
\hline $\begin{array}{l}\text { 47.- La Ciencia ha evolucionado históricamente } \\
\text { mediante la acumulación sucesiva de las teorías } \\
\text { verdaderas. }\end{array}$ & 164 & 3,10 & ,970 & 136 & 2,76 & 1,368 \\
\hline $\begin{array}{l}\text { 51.- Las hipótesis dirigen el proceso de investigación } \\
\text { científica. }\end{array}$ & 163 & 3,82 & ,731 & 135 & 3,84 & 848 \\
\hline $\begin{array}{l}\text { 55.- La experimentación se utiliza en ciertos tipos de } \\
\text { investigación científica mientras que en otras no. }\end{array}$ & 162 & 2,86 & 1,068 & 136 & 2,77 & 1,229 \\
\hline
\end{tabular}




\begin{tabular}{|c|c|c|c|c|c|c|}
\hline Modelo Didáctico & \multicolumn{3}{|c|}{ PRE MAES } & \multicolumn{3}{|c|}{ POST MAES } \\
\hline Declaraciones & $\mathrm{N}$ & $\bar{X}$ & $\bar{\sigma}$ & $\mathrm{N}$ & $\bar{X}$ & $\sigma$ \\
\hline $\begin{array}{l}\text { 2.- El profesor o la profesora, al programar, debe } \\
\text { planificar con todo detalle las tareas a realizar por el } \\
\text { o ella y por los alumnos/ alumnas, para evitar la } \\
\text { improvisación. }\end{array}$ & 165 & 3,53 & 1,107 & 140 & 3,67 & 1,115 \\
\hline $\begin{array}{l}\text { 3.- La Didáctica se considera, en la actualidad, una } \\
\text { disciplina científica. }\end{array}$ & 163 & 3,39 & ,952 & 137 & 3,64 & 1,063 \\
\hline $\begin{array}{l}\text { 12.- La Didáctica debe definir normas y principios } \\
\text { que guíen y orienten la práctica educativa. }\end{array}$ & 164 & 3,98 & ,763 & 137 & 3,99 & ,776 \\
\hline $\begin{array}{l}\text { 13.- Los profesores y profesoras deben hacer } \\
\text { compatibles las tareas de enseñanza con las de } \\
\text { investigación de los procesos que se dan en su } \\
\text { clase. }\end{array}$ & 165 & 4,03 & ,728 & 138 & 4,12 &, 820 \\
\hline $\begin{array}{l}\text { 16.- Los procesos de enseñanza - aprendizaje que } \\
\text { se dan en cada clase son fenómenos complejos en } \\
\text { los que intervienen innumerables factores. }\end{array}$ & 165 & 4,16 & ,715 & 139 & 4,30 & ,804 \\
\hline $\begin{array}{l}\text { 17.- Los alumnos y alumnas no deben intervenir } \\
\text { directamente en la programación y evaluación de la } \\
\text { actividad de su clase. }\end{array}$ & 165 & 2,44 & 1,160 & 139 & 2,10 & 1,092 \\
\hline $\begin{array}{l}\text { 18.- La Didáctica pretende describir y comprender } \\
\text { los procesos de enseñanza/aprendizaje que se dan } \\
\text { en las aulas. }\end{array}$ & 165 & 3,81 & ,801 & 137 & 3,87 & ,821 \\
\hline $\begin{array}{l}\text { 20.- Los objetivos, organizados y jerarquizados } \\
\text { según su grado de dificultad, deben ser el } \\
\text { instrumento esencial que dirija la práctica educativa. }\end{array}$ & 164 & 3,48 & ,896 & 135 & 3,42 & 1,054 \\
\hline $\begin{array}{l}\text { 26.- La organización de los centros de enseñanza } \\
\text { debe basarse en agrupamientos y horarios flexibles. }\end{array}$ & 164 & 3,47 & ,923 & 137 & 3,55 & ,899 \\
\hline $\begin{array}{l}\text { 29.- El objetivo básico de la Didáctica es definir las } \\
\text { técnicas más adecuadas para una enseñanza de } \\
\text { calidad. }\end{array}$ & 164 & 3,90 &, 711 & 136 & 3,87 & 901 \\
\hline $\begin{array}{l}\text { 30.- El trabajo en el aula debe estar organizado } \\
\text { fundamentalmente en torno a los contenidos del } \\
\text { área. }\end{array}$ & 164 & 3,63 & ,836 & 136 & 3,18 & ,921 \\
\hline $\begin{array}{l}\text { 31.- La evaluación debe centrarse en medir el nivel } \\
\text { alcanzado por los alumnos/alumnas respecto a los } \\
\text { objetivos previstos. }\end{array}$ & 163 & 3,48 & ,939 & 137 & 3,36 & 1,090 \\
\hline $\begin{array}{l}\text { 34.- Un buen libro de texto es un recurso } \\
\text { indispensable para la enseñanza de las ciencias. }\end{array}$ & 164 & 3,10 & 1,168 & 137 & 2,58 & 1,136 \\
\hline $\begin{array}{l}\text { 53.- La Didáctica se desarrolla mediante procesos } \\
\text { de investigación teórico - prácticos. }\end{array}$ & 164 & 3,82 & ,717 & 135 & 3,79 & ,811 \\
\hline
\end{tabular}




\begin{tabular}{|c|c|c|c|c|c|c|}
\hline Metodología del Profesor & \multicolumn{3}{|c|}{ PRE MAES } & \multicolumn{3}{|c|}{ POST MAES } \\
\hline Declaraciones & $\mathrm{N}$ & $\bar{X}$ & $\sigma$ & $\mathrm{N}$ & $\bar{X}$ & $\sigma$ \\
\hline $\begin{array}{l}\text { 1.- Los alumnos y las alumnas aprenden } \\
\text { correctamente los conceptos científicos cuando } \\
\text { realizan actividades prácticas. }\end{array}$ & 163 & 4,15 & ,705 & 140 & 3,78 & 823 \\
\hline $\begin{array}{l}\text { 6.- La realización de problemas en clase es la mejor } \\
\text { alternativa al método magistral de enseñanza de las } \\
\text { ciencias. }\end{array}$ & 165 & 3,59 & ,789 & 139 & 3,32 & 1,030 \\
\hline $\begin{array}{l}\text { 7.- La manera correcta de aprender ciencias en la } \\
\text { Enseñanza Secundaria Obligatoria es aplicando el } \\
\text { método científico en el aula. }\end{array}$ & 165 & 3,38 &, 822 & 137 & 3,51 & 2,744 \\
\hline $\begin{array}{l}\text { 9.- El método de enseñanza es la manera de dar los } \\
\text { contenidos científicos. }\end{array}$ & 165 & 3,39 & ,934 & 134 & 3,01 & 1,100 \\
\hline $\begin{array}{l}\text { 10.- La biblioteca y el archivo de clase son recursos } \\
\text { imprescindibles para la enseñanza de las ciencias. }\end{array}$ & 165 & 3,73 & ,905 & 138 & 3,51 & 882 \\
\hline $\begin{array}{l}\text { 15.- El profesor o profesora debe sustituir el } \\
\text { temario por un listado de problemas más o menos } \\
\text { abiertos que abarquen los mismos contenidos. }\end{array}$ & 165 & 2,84 & ,987 & 139 & 3,12 & 1,097 \\
\hline $\begin{array}{l}\text { 25.- El contacto con la realidad y el trabajo en el } \\
\text { laboratorio son imprescindibles para el aprendizaje } \\
\text { científico. }\end{array}$ & 164 & 4,19 & ,772 & 138 & 4,28 & ,783 \\
\hline $\begin{array}{l}\text { 36.- Cada profesor o profesora construye su propia } \\
\text { metodología para la enseñanza de las ciencias. }\end{array}$ & 164 & 3,73 & ,816 & 137 & 3,77 & ,939 \\
\hline $\begin{array}{l}\text { 37.- Los métodos de enseñanza de las ciencias } \\
\text { basados en la investigación del alumno/alumna no } \\
\text { provocan el aprendizaje de contenidos concretos. }\end{array}$ & 164 & 2,15 & 954 & 136 & 2,01 & 1,018 \\
\hline $\begin{array}{l}\text { 43.- Para enseñar ciencias es necesario explicar } \\
\text { detenidamente los temas para facilitar el } \\
\text { aprendizaje de los alumnos y alumnas. }\end{array}$ & 164 & 3,49 & 833 & 136 & 2,85 & ,993 \\
\hline $\begin{array}{l}\text { 45.- El aprendizaje de las ciencias basado con el } \\
\text { trabajo con el libro de texto no motiva a los } \\
\text { alumnos y alumnas. }\end{array}$ & 164 & 3,47 & ,930 & 137 & 3,59 & ,989 \\
\hline $\begin{array}{l}\text { 49.- En la clase de ciencias es conveniente que los } \\
\text { alumnos / alumnas trabajen formando equipos. }\end{array}$ & 164 & 3,77 & 904 & 136 & 3,90 & ,782 \\
\hline $\begin{array}{l}\text { 52.- La mayoría de los libros de texto sobre ciencias } \\
\text { experimentales no facilitan la comprensión y el } \\
\text { aprendizaje de los alumnos/alumnas. }\end{array}$ & 163 & 3,10 & 991 & 136 & 3,33 & 1,061 \\
\hline $\begin{array}{l}\text { 56.- La enseñanza de las ciencias basada en la } \\
\text { explicación verbal de los temas favorece que el } \\
\text { alumno/alumna memorice mecánicamente el } \\
\text { contenido. }\end{array}$ & 162 & 2,73 & 1,130 & 136 & 2,57 & 1,297 \\
\hline
\end{tabular}




\section{ANEXO II. Características de los distintos Modelos Didácticos}

(Reelaborado a partir de Porlán y Martín, 1991, Porlán y otros, 1996, Porlán y Rivero, 1998 y García P., 2000)

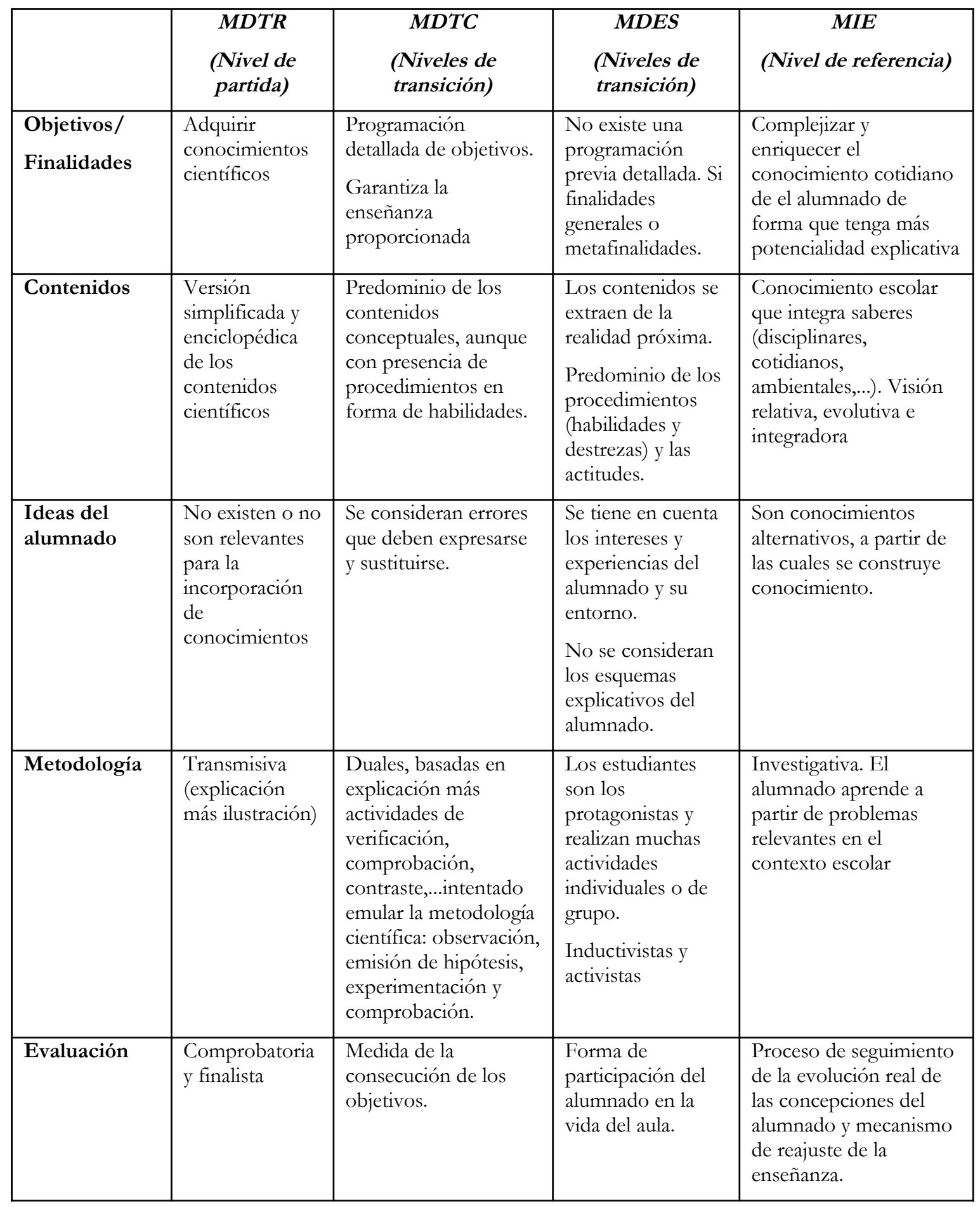

